\title{
Investigation of implantable signal transmission characteristics based on visible data of the human leg
}

\author{
Yue-Ming Gao ${ }^{1,2^{*}}$, Yan-Ting Ye ${ }^{1,2}$, Shi Lin ${ }^{1,2}$, Željka Lučev Vasić ${ }^{3}$, Mang-I Vai ${ }^{2,4,5}$, Min Du ${ }^{1,2}$, Mario Cifrek ${ }^{3}$ \\ and Sio-Hang Pun ${ }^{4}$
}

*Correspondence:

fzugym@163.com

${ }^{1}$ College of Physics

and Information Engineering,

Fuzhou University,

Fuzhou 350116, China

Full list of author information

is available at the end of the article

\begin{abstract}
Background: Signal transmission characteristics between implanted medical devices and external equipment has been a common key issue, as has the problem of supplying energy to the devices. It can be used to enable signal transmission from implanted devices that the human body's conductive properties. Using signal transmission by galvanic coupling is one of the most effective signal transmission methods.
\end{abstract}

Methods: The signal transmission characteristics by galvanic coupling of implantable devices using a frequency range of $10 \mathrm{kHz}$ to $1 \mathrm{MHz}$ was analyzed in this article. A finite element (FEM) model and a phantom model established by visible human leg data were used to investigate the signal transmission characteristics of implant-to-surface, with implantable receiver electrodes at different locations.

Results: The results showed that the FEM model and the phantom model had similar implantable signal transmission characteristics, with an increase of frequency, signal attenuation basically remained unchanged. The gain in signal attenuation in the fixed attenuation values fluctuated no more than $5 \mathrm{~dB}$ and signal attenuation values rose as the channel length increased.

Conclusions: Our results of signal transmission characteristics of surface-to-implant will provide a theoretical basis for implantable transceiver design, and for realization of a recharging method for implanted medical devices.

Keywords: Channel modeling, Finite element model, Implantable signal transmission, Phantom model

\section{Background}

Implanted medical devices enable long-term continuous monitoring of physiological changes, diagnosis and analysis of some chronic diseases, and control of organ feature loss. They also enable more comprehensive health monitoring [1-4]. Signal transmission between those devices and external equipment has been a common key issue, as has the problem of supplying energy to the devices. It can be used to enable signal transmission from implanted devices that the human body's conductive properties. This is one of the most effective signal transmission methods that using signals transmission by galvanic coupling, because it does not have to design the antenna of the transceiver, yet the power 
consumption maybe further decrease. Moreover, the surface-to-implant recharging of implanted medical devices become possible [5], since the path loss of the signal transmission is not very high.

Several research groups have investigated signal transmission from implantable devices in the human body. Lindsey et al. [6] implanted a sensor in a cadaver knee joint and measured the tension of the ligaments from the surface of the leg. Experimental results show that for signal transmission from implant to surface, signal attenuation is closely related to the amount of current, the size of the surface electrode, and the distance between the electrodes. Wegmueller et al. [7] designed a system that could achieve signal transmission from implant to implant. They experimented with the electrical properties of emulated muscle. Shiba et al. studied signal transmission from human tissue to the body surface, verifying the feasibility of implant-to-surface signal transmission [8]. Ferguson et al. [9] described and compared several methods of signal transmission using the conductive properties of the body between implanted medical devices. In terms of energy transmission, $[10,11]$ analyzed the feasibility of using volume conduction for energy transfer. A transcutaneous battery recharging circuit unit was designed that takes advantage of skin volume conduction. On that basis, a low-power digital communication model based on volume conduction was presented.

Most of the aforementioned studies of implant signal transmission using the conductive properties of the body were carried out in physiological saline or other liquid that had the electrical properties of human tissues. However, that approach cannot emulate the complex anatomical and geometrical characteristics of the human body, and leads to experimental results not completely consistent with those in realistic. Most research has concentrated on verifying the feasibility of implant signal transmission; research on the characteristics of the transmission is scarce. We investigated those characteristics to better understand effective signal transmission and to offer a theoretical analysis of the recharging of implanted medical devices. In this research, surface-to-implant signal transmission characteristics in a frequency range from $10 \mathrm{kHz}$ to $1 \mathrm{MHz}$ was analyzed using a finite element method (FEM) model and a phantom model. We established an implantable FEM model using visible human leg data and FEM method. We then synthesized a material whose dielectric properties were similar to those of human tissues. An implantable phantom model was made using the material and a visible human leg outer contour. Signal transmission characteristics from the surface to the implant were analyzed by doing implant experiments in a FEM model and a phantom model. The mutual complementation and authentication of the FEM and phantom models lent veracity and reliability to the implantable signal transmission characteristics studied in this article. Our results will provide a theoretical basis for implantable transceiver design, and for realization of a recharging method for implanted medical devices.

\section{Methods}

\section{The implantable leg FEM model}

In the human legs, the tissues of fat, muscle and bone accounted for a larger volume, so that the influence of fat, muscle and bone on signal transmission cannot be ignored. And the skin is directly contacted with the electrode, the ion current and the electron current can be exchanged on the surface of the skin-electrode [12]. It plays an important role in 
the signal transmission process of surface-to-implant. Therefore, in this article, neglecting other small tissues such as blood vessels, lymph, mucous membrane, the human leg model was divided into four layers of skin, fat, muscle and bone [13].

Thus, a four-layer leg model was reconstructedusing transverse anatomical fault images of a male provided by the U.S. National Library of Medicine and the University of Colorado. First, the leg structure is divided into several different tissues, including skin, fat, muscle and bone. Next, the outline of each tissue layers on every anatomical image are extracted automatically, and the contour line of tissues are reconstructed through three-dimensional reconstruction software GEOMAGIC Studio (GEOMAGIC Inc., North Carolina, USA). Finally, using SOLIDWORKS (Dassault Systemes S.A., Massachusetts, USA) to fill each layer with the respective tissues to acquire a personalized leg model [14].

The four-layer leg model included skin, fat, muscle and bone layers, as shown in Fig. 1. The complex frequency behavior of the dielectric properties of human tissue; that is, conductivity $\sigma$ and permittivity $\varepsilon$, were derived from the parametric modes of Gabriel [15]. The FEM simulation is conducted in the COMSOL MULTIPHYSICS 5.2 (COMSOL Inc., Stockholm, Sweden).
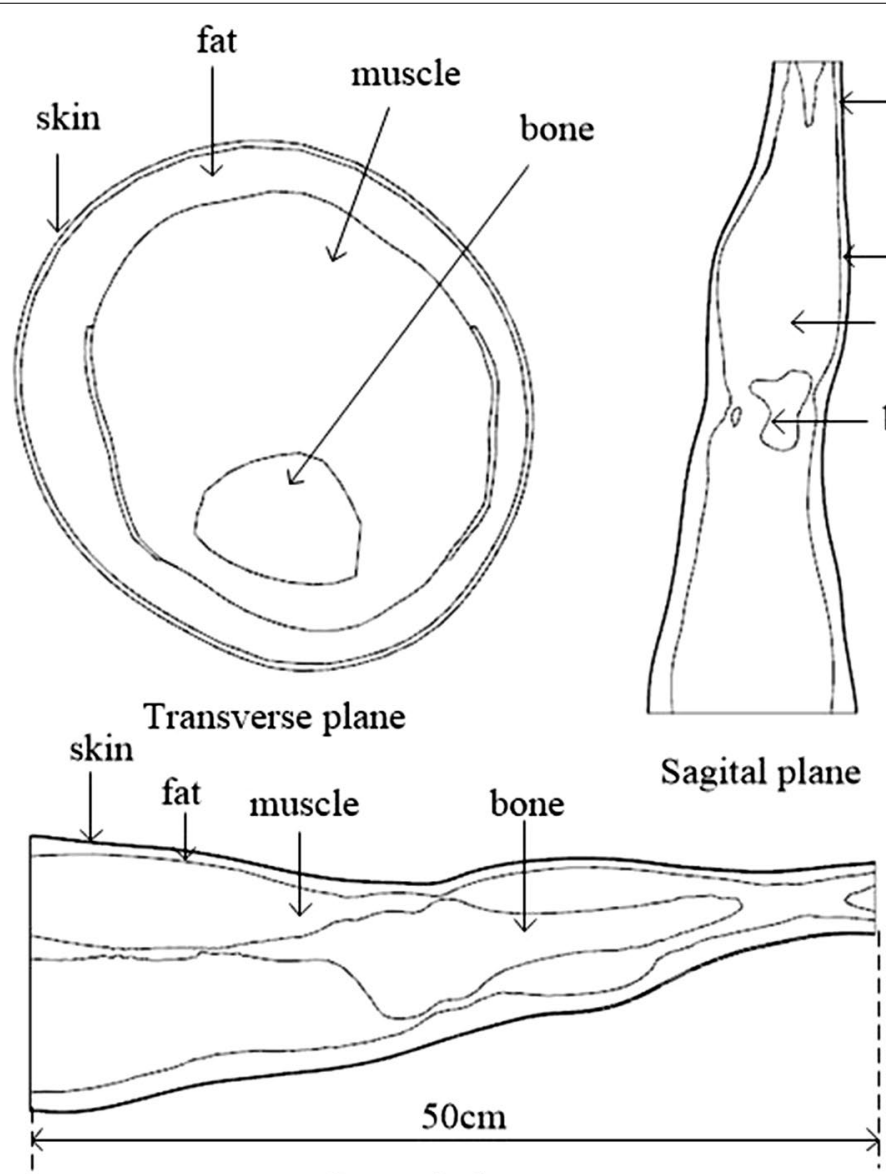

Coronal plane

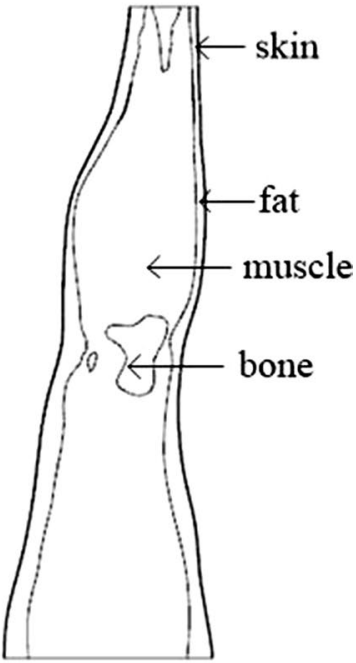

Sagital plane

Fig. 1 Three views of the human body leg model 
In an implantable signal transmission system (Fig. 2), a pair of transmitter electrodes was attached to the skin surface so the voltage or current signal could pass into the body [16]. A pair of implantable receiver electrodes was attached at the junction of the muscle and fat layers to receive electrical signals. The electrode dimensions were $4 \mathrm{~cm} \times 4 \mathrm{~cm}$, $1 \mathrm{~mm}$ thick, with a conductivity of $6 \times 103 \mathrm{~S} / \mathrm{m}$ and a relative dielectric constant of 1 .

To ensure close contact of the electrodes with human tissue, we positioned the surface transmitter electrodes and implantable receiver electrodes in the following ways: The contour of the skin layer to the external upset was $1 \mathrm{~mm}$ and made a Boolean intersection with a rectangular parallelepiped whose cross section was $4 \mathrm{~cm} \times 4 \mathrm{~cm}$, to obtain a transmitter electrode that was in close contact with the skin surface. Voltage or current passed into the body through the transmitter electrodes. Similarly, the contour of the muscle layer to the external upset was $1 \mathrm{~mm}$ and made a Boolean intersection with a rectangular parallelepiped whose cross section was $4 \mathrm{~cm} \times 4 \mathrm{~cm}$, to obtain an implantable receiver electrode that was in close contact with the muscle layer. The surface transmitter electrodes and implantable receiver electrodes shown in Fig. 3.

Based on the geometry model of the human leg (Fig. 1), the mechanism of the implantable signal transmission was investigated at modes A, B, and C, as described in "Simulation mode setups". Because the total charge density in a human body is zero, a Maxwell equation could be equivalent to the Laplace equation [17-19].

$$
-\nabla \times\left[\left(\sigma+i \omega \varepsilon_{0} \varepsilon_{i}\right) \nabla V\right]=0
$$

where $\sigma$ is the electrical conductivity, $\omega$ is the angular frequency, $\varepsilon_{0}$ is the permittivity of free space, $\varepsilon_{\mathrm{i}}$ is the permittivity of the medium, and $V$ is the potential difference of the body surface.

The input electrical signal at the surface transmitter electrodes was

$$
V=V_{0}
$$

where $V_{0}$ was the amplitude of the voltage injected into the limb.

Current and voltage continuity conditions of the receiving electrodes and of the surface of the human model were given by:

$$
\left\{\begin{array}{c}
V_{l-1}=V_{l} \\
J_{l-1}=J_{l}
\end{array}\right.
$$

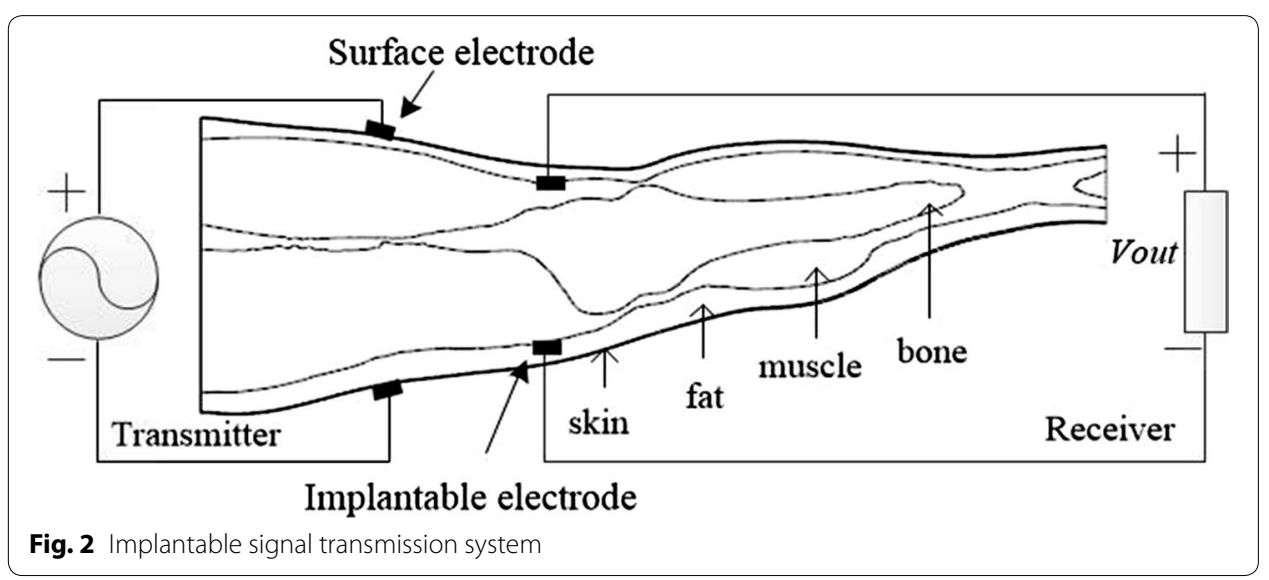



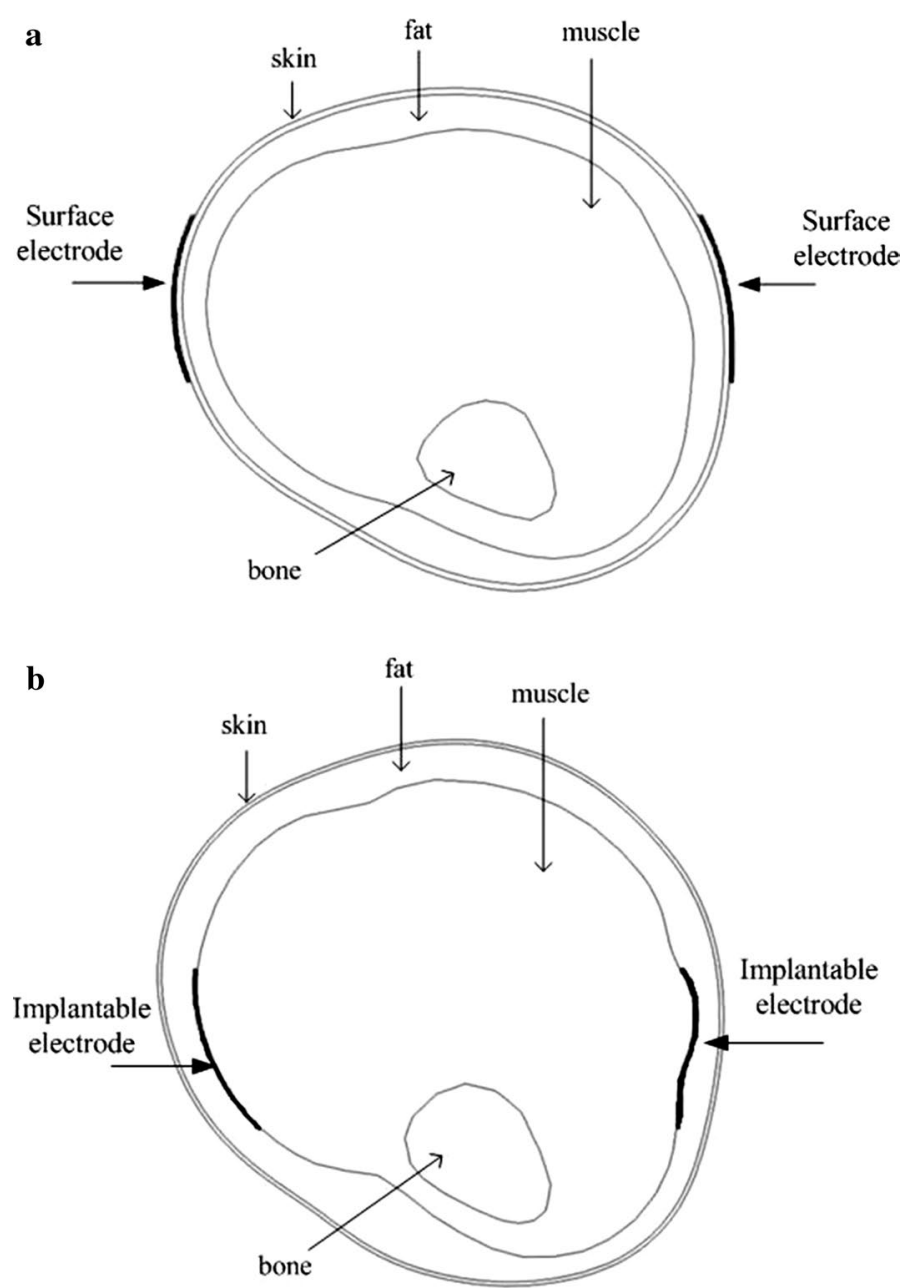

Fig. 3 The transverses plane of electrode. a Surface transmitter electrodes. b Implantable receiver electrodes

where, $J_{l-1}, J_{l}$ and $V_{l-1}, V_{l}$ indicate the current density and the voltage of the adjacent tissues, and $l$ indicates the tissue layer.

Thus, a default fine free tetrahedral element is used to mesh the human body leg model. The solution for the model is obtained by running the direct solver PARDISO in a fully coupled manner.

\section{Simulation mode setups}

In accordance with the location of the implantable receiver electrodes, simulation devices were divided into the following three modes:

\section{Mode A}

As shown in Fig. 4, the receiver electrodes were implanted in the calf $5 \mathrm{~cm}$ from the bottom of the leg model at the junction of the fat and muscle layers. The transmitter electrodes were placed on the surface of the skin layer. Transmitter and receiver electrodes were separated by 6 and $10 \mathrm{~cm}$. 

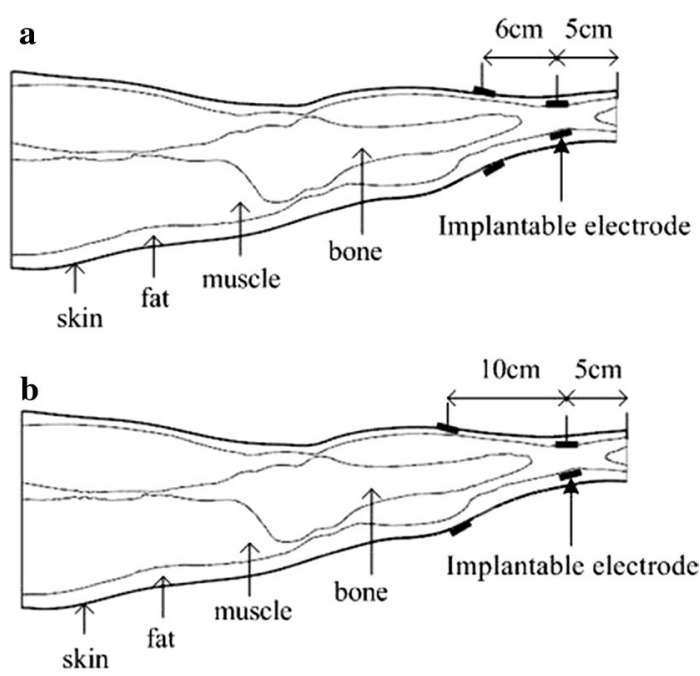

Fig. 4 Mode A: Implantable receiver electrode placement at the calf. a Transmitter and receiver electrodes separated by $6 \mathrm{~cm}$. b Electrodes separated by $10 \mathrm{~cm}$

\section{Mode B}

As shown in Fig. 5, the receiver electrodes were implanted near the knee $30 \mathrm{~cm}$ from the bottom of the leg model at the junction of the fat and muscle layers. The transmitter electrodes were placed on the surface of the skin layer. Mode B1: Transmitter and receiver electrodes were separated by $6 \mathrm{~cm}$ (Fig. 5a) and $10 \mathrm{~cm}$ (Fig. 5b) toward the direction of the calf. Mode B2: Transmitter and receiver electrodes were separated by $6 \mathrm{~cm}$ (Fig. 5c) and $10 \mathrm{~cm}$ (Fig. 5d) toward the direction of the thigh.

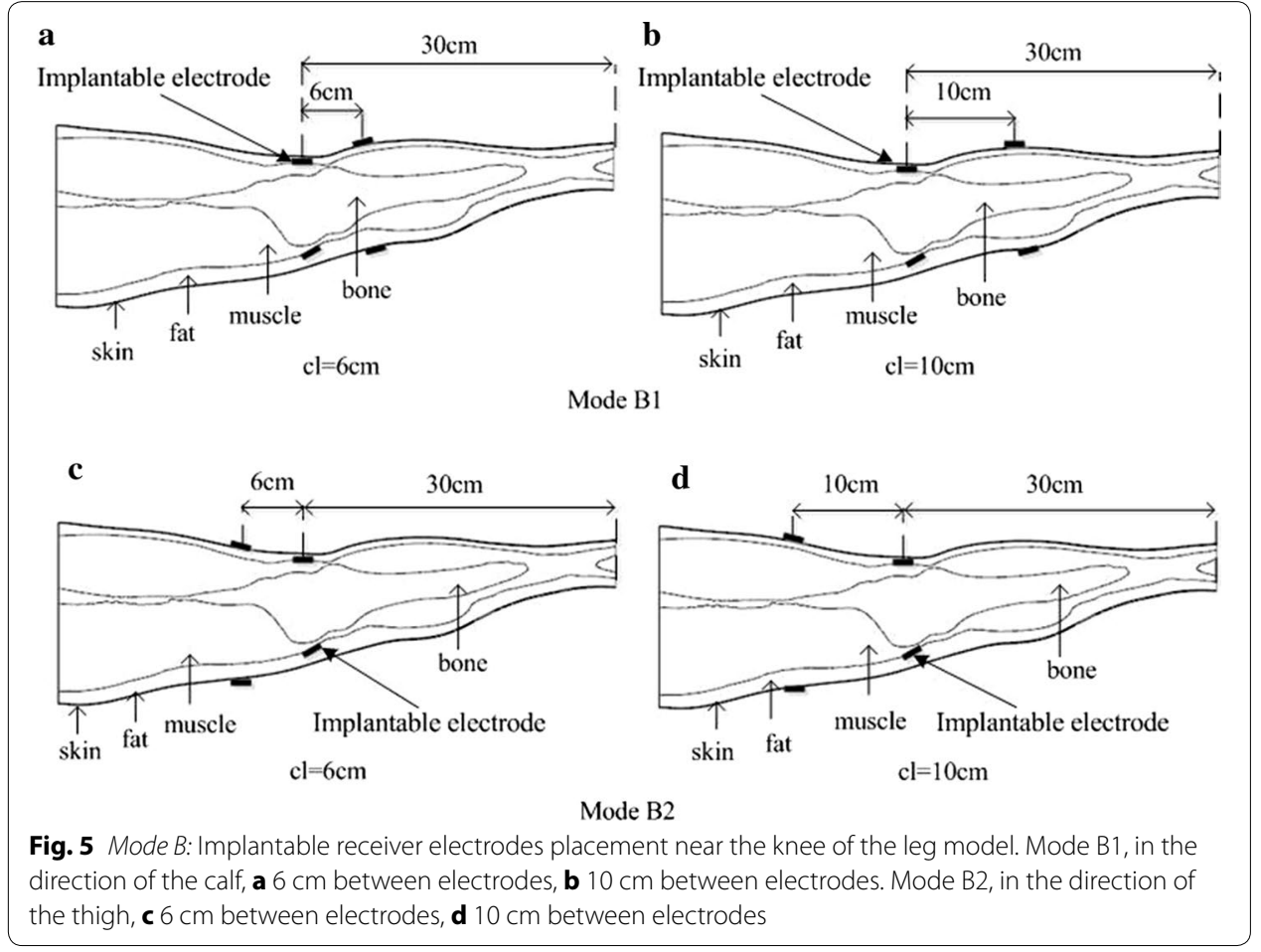




\section{Mode C}

As shown in Fig. 6, the receiver electrodes were implanted at the thigh $45 \mathrm{~cm}$ from the bottom of the leg model at the junction of fat and muscle layers. The transmitter electrodes were placed on the surface of the skin layer. Transmitter and receiver electrodes were separated by $6 \mathrm{~cm}$ and $10 \mathrm{~cm}$.

\section{The implantable leg phantom model}

The FEM fully takes into account the characteristics of human anatomy and can describe the distribution of electromagnetic fields in the organism, but its shortage was not suitable for the actual performance test. An implant phantom model consists of synthetic material whose dielectric properties are similar to those of human tissues. Using this model to emulate the human body enables more realistic experimentation. The FEM and phantom models we used enabled research of the channel characteristics of implantable signal transmission to be more realistic, accurate, and reliable.

The greatest influence on signal transmission through the human body by galvanic coupling are the skin, fat, and muscle layers $[19,20]$. The effects of the periosteum and bone marrow are minor [15], so the influence of bones can be ignored. As shown in Table 1, it can be finding that the conductivity of the fat and skin layers is almost the same, when the frequency is near $40 \mathrm{kHz}$ [16], so they are equivalent to a single layer. The phantom model was divided into muscle and skin-fat layers.

The human leg contour model was rebuilt using visible human leg data, and then the outer contour molds of the muscle and skin-fat layers were printed by a 3D printer, as shown in Fig. 7.

Agar, potassium chloride, hydroxyethyl cellulose (HEC) and distilled water were mixed as shown in Table 2 to emulate human tissue. The electrical conductivity of the leg
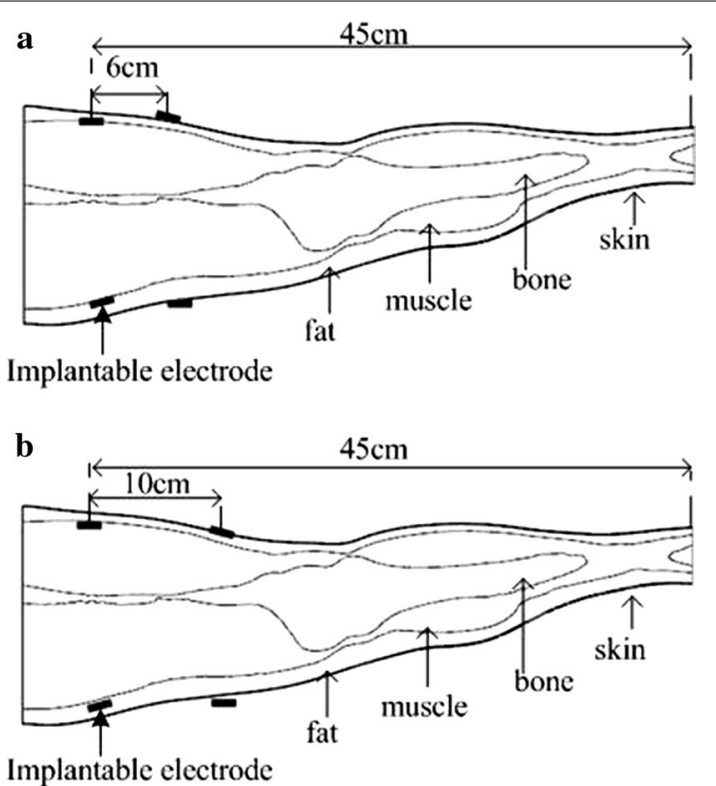

Fig. 6 Mode C: Implantable receiver electrode placement at the thigh. a $6 \mathrm{~cm}$ between electrodes. $\mathbf{b} 10 \mathrm{~cm}$ between electrodes 
Table 1 Conductivity of each human body layer at different frequencies

\begin{tabular}{|c|c|c|c|c|}
\hline \multirow[t]{2}{*}{ Conductivity (S/m) } & \multicolumn{4}{|c|}{ Frequency (Hz) } \\
\hline & 10,000 & 40,000 & 70,000 & 100,000 \\
\hline Muscle & 0.34083 & 0.34977 & 0.35579 & 0.36185 \\
\hline Fat & 0.00293 & 0.02167 & 0.04475 & 0.06583 \\
\hline Wet skin & 0.02383 & 0.02419 & 0.02433 & 0.02441 \\
\hline
\end{tabular}
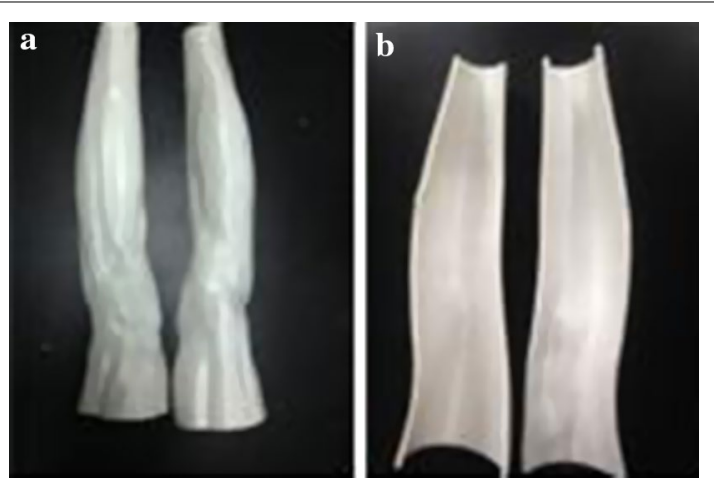

Fig. 7 3D-printed outer contour molds of human legs. a Outer contour mold of muscle layer (outside layer). b Outer contour mold of skin-fat layer (inside layer)

Table 2 Proportions of ingredients to emulate human muscle and skin-fat layers

\begin{tabular}{llllll}
\hline Tissue & \multicolumn{2}{l}{ Ingredient } & & & \\
\cline { 2 - 6 } & Agar $(\mathbf{g})$ & $\mathbf{K C l}(\mathbf{g})$ & HEC $(\mathbf{g})$ & Distilled water $(\mathbf{m L})$ & Methylene blue \\
\hline Skin-fat & 40 & 0.1652 & 80 & 1400 & Appropriate \\
Muscle & 40 & 2.8 & 80 & 1400 & None \\
\hline
\end{tabular}

model was changed by adjusting the quantity of potassium chloride to approximate the conductivities of muscle and skin-fat layers.

After the muscle layer of the phantom model was produced, we put receiver electrodes in fixed positions at the surface of the layer according to modes A, B, and C. Then we covered the muscle layer with the outer skin-fat layer. During the latter's production we added a biological stain called methylene blue to distinguish the two layers (Fig. 8).

Figure 9 is a block diagram of the galvanic coupling implantable signal transmission experiment in the leg phantom model. A CXA Agilent N9000 spectrum analyzer was used in the experiment of galvanic coupling implantable signal transmission in the phantom model. A $0 \mathrm{dBm}$ output signal from the extremity of the TX was injected into the leg model through the transmitting electrodes. The receiving signal was detected by a differential probe in order to avoid the results being affected by the human body and the ground connecting to form a looping-in, and then voltage gain was shown on the screen.

The voltage gain was used to represent the channel attenuation characteristics, with their expression shown in Eq. (4):

$$
\operatorname{Gain}(\mathrm{d} B)=20 \cdot \log \left(V_{\mathrm{r}} / V_{\mathrm{t}}\right)
$$




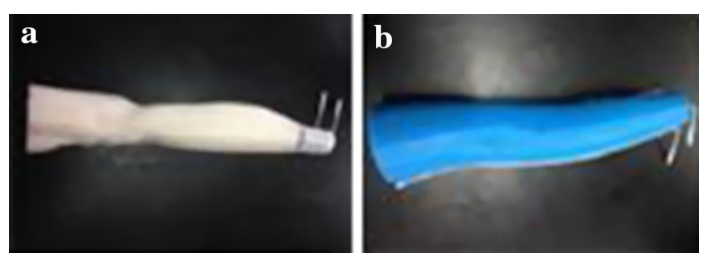

Fig. 8 Phantom model of mode A. a Muscle layer. b Complete model of mode A showing outer skin-fat layer

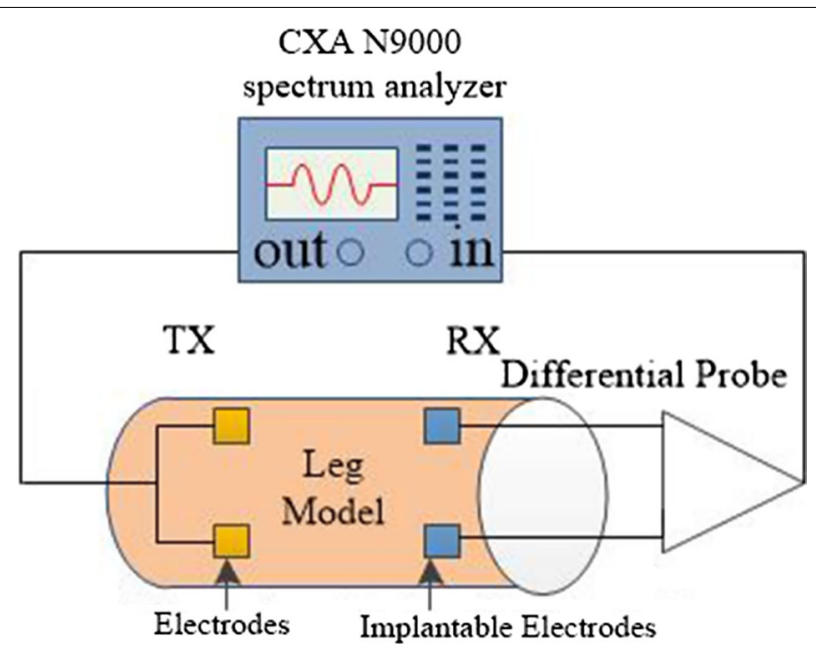

Fig. 9 Galvanic coupling implantable signal transmission experiment in the phantom model

where Gain are the channel attenuation characteristics in $\mathrm{dB}, V_{\mathrm{r}}$ is the receiving voltage, and $V_{t}$ is the transmitting voltage.

\section{Results}

As described in "Methods", the simulation devices had three modes: A, B, and C. The channel characteristics of the implantable signal transmission of the FEM and phantom models were investigated at each of the modes.

Figure 10 shows the results when the implantable receiver electrodes were placed in the calf (mode A). The FEM model signal variation was consistent with that of the phantom model, with little change in the increase of frequency. The gain in a fixed attenuation value fluctuation was no more than $5 \mathrm{~dB}$. Figure 10 also shows that there was higher attenuation values with the channel length increased. For either channel length $(\mathrm{cl}=6 \mathrm{~cm}$ and $\mathrm{cl}=10 \mathrm{~cm})$, the errors between the FEM model and the phantom model were limited to $8 \mathrm{~dB}$. When $\mathrm{cl}=6 \mathrm{~cm}$, the phantom model signal gain was less than that of the FEM model, and when $\mathrm{cl}=10 \mathrm{~cm}$, the FEM model signal attenuation was less than that of the phantom model

Shown in Figs. 11 and 12, with the implantable receiver electrodes placed at the middle of the leg model (mode B), it could be found that with increasing frequency the signal gain changed little, and the FEM and phantom models had similar channel characteristics. Comparing mode B1 (Fig. 11) with mode B2 (Fig. 12), with transmitter electrodes 

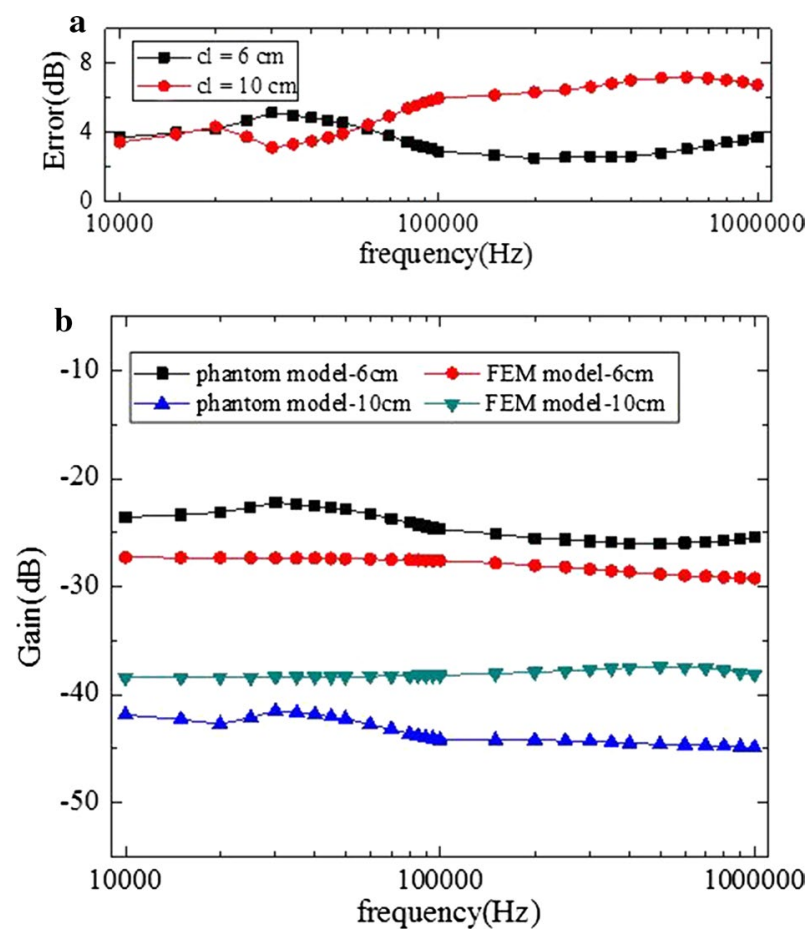

Fig. 10 In mode A, a comparison between implantable FEM model simulation results and phantom model experiment results. a Error of the phantom and FEM models. $\mathbf{b}$ Results of the phantom model experiment and the FEM model simulation

placed in the direction of thigh or calf, the basic characteristics of the channel were changeless.

As shown in Fig. 13, with the implantable receiver electrodes placed in the thigh (mode $\mathrm{C}$ ), we found that similar to A and B modes the FEM and phantom models had similar channel characteristics, with signal attenuation changeless in the frequencies of $10 \mathrm{kHz}$ to $1 \mathrm{MHz}$. For the phantom model, when $\mathrm{cl}=6 \mathrm{~cm}$, the signal attenuation with the implantable receiver electrodes placed in the calf (mode A) and the thigh (mode $\mathrm{C}$ ) was approximately $25 \mathrm{~dB}$, which was less than in mode $\mathrm{B}$. When $\mathrm{cl}=10 \mathrm{~cm}, \mathrm{~B} 1$ mode and $C$ mode channel characteristic were approximately $35 \mathrm{~dB}$, which were better than that of the other modes. For the FEM model, when $\mathrm{cl}=6 \mathrm{~cm}$, the signal attenuation with the implantable receiver electrodes placed in the calf (mode A) and the thigh (mode $\mathrm{C}$ ) was approximately $27 \mathrm{~dB}$, which was less than that of mode $\mathrm{B}$. When $\mathrm{cl}=10 \mathrm{~cm}$, compared with other modes channel characteristics were better in modes B1 and C.

According to the experiment results of the FEM and phantom models, we found that when $\mathrm{cl}=6 \mathrm{~cm}$ in the frequencies of $10 \mathrm{kHz}-1 \mathrm{MHz}$, with the implantable receiver electrodes placed in the calf (mode A) and the thigh (mode $\mathrm{C}$ ) the signal attenuation of the phantom model was less than that of the FEM model. In mode B (near the knee), the signal attenuation of the FEM model was less than that of the phantom mode. Finally, when $\mathrm{cl}=10 \mathrm{~cm}$, the FEM signal attenuation was less than that of the phantom model in modes A, B, and C. 

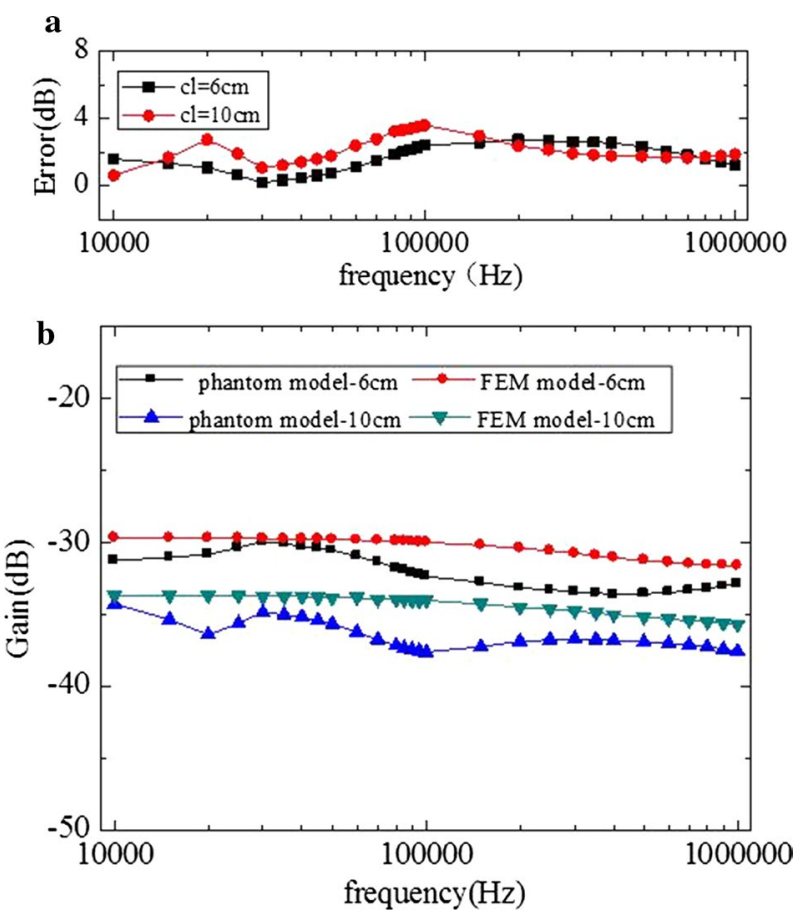

Fig. 11 In mode B1, in the direction of the calf, a comparison between implantable FEM model simulation results and phantom model experiment results. a Error of the phantom model and FEM model. b Results of phantom model experiment and FEM model simulation
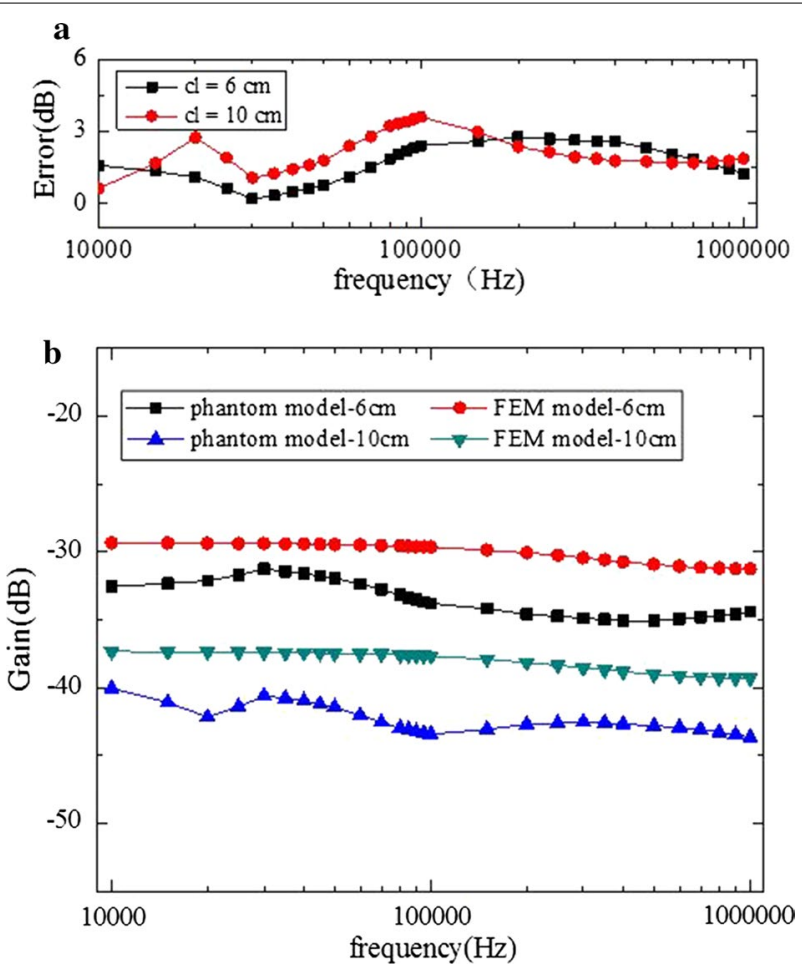

Fig. 12 In mode B2, in the direction of the thigh, a comparison between implantable FEM model simulation results and phantom model experiment results. a Error of the phantom model and FEM model. b Results of phantom model experiment and FEM model simulation 

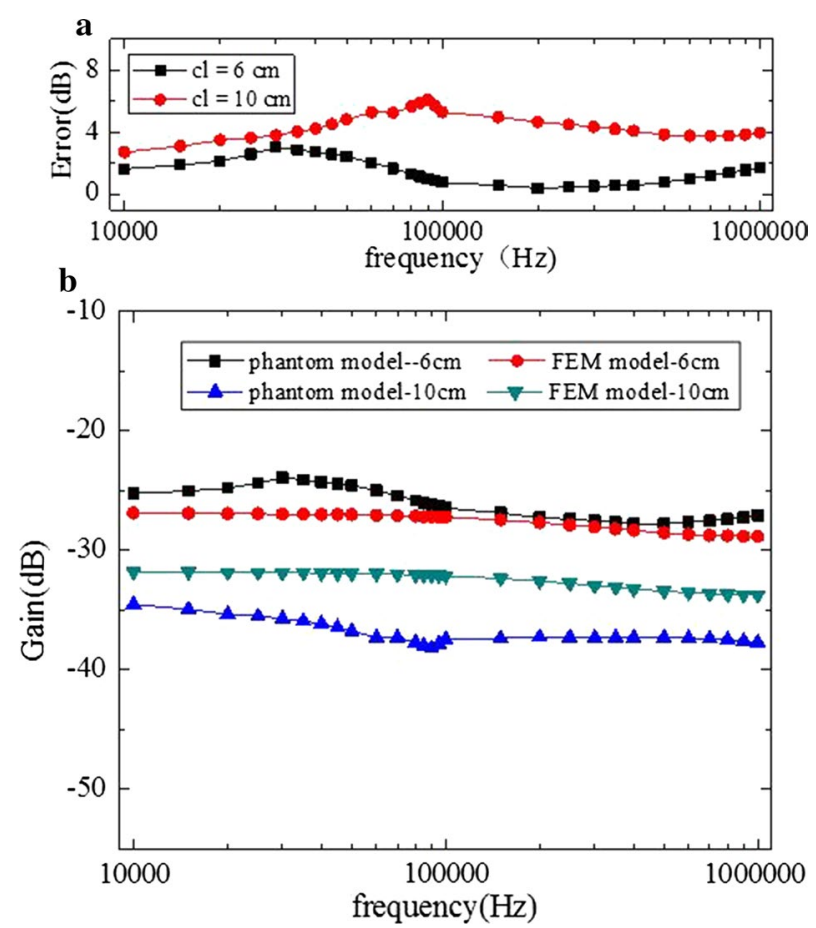

Fig. 13 In mode C, a comparison between the implantable FEM model simulation results and the phantom model experiment results. a Error of the phantom and FEM models. $\mathbf{b}$ Results of the phantom model experiment and the FEM model simulation

\section{Discussion}

An implantable FEM model and a phantom model using visible human leg data were created to study surface-to-implant implantable signal transmission characteristics in the frequency range from $10 \mathrm{kHz}$ to $1 \mathrm{MHz}$ at modes $\mathrm{A}, \mathrm{B}$, and $\mathrm{C}$ in this article. The results showed that the relationship between the voltage attenuation and the frequency of the surface-to-implant signal transmission path was consistent at modes $\mathrm{A}, \mathrm{B}$, and $C$ with an increase in frequency, signal attenuation remained basically unchanged. The gain in the fixed attenuation values fluctuated no more than $5 \mathrm{~dB}$, and increasing the channel length increased signal attenuation. It reveals that, in the frequency range of $10 \mathrm{kHz}$ to $1 \mathrm{MHz}$, the main factors affecting signal transmission characteristics of surface-to-implant was not the frequency, and channel length will be a key parameter to affect the efficiency of power supply of implantable medical devices. In the future, the $10 \mathrm{kHz}$ to $1 \mathrm{MHz}$ frequency range can be used as the working frequency of implantable devices to realize the power supply in body surface. Besides, and in order to get better signal transmission effect and improve the power supply efficiency, the channel lengths of surface-to-implant signal transmission should be shorten as much as possible. The A, $B$ and $C$ modes had the same results, which indicate that the position of the implantable electrode has little effect on the channel characteristics of surface-to-implant signal transmission. The errors of A, B and C was mainly caused by the geometry difference of each tissue layer of the human leg.

The errors of the implantable FEM model and the phantom model were a maximum of $8 \mathrm{~dB}$ at modes $\mathrm{A}, \mathrm{B}$, and $\mathrm{C}$, with those of the implantable phantom model 
basically equivalent to those of the implantable FEM model. The cause of the errors of the implantable FEM model and the phantom model was due to the FEM model including four layers of skin, fat, muscle and bone. However, the production process of phantom model ignored the influence of the bone layer, and the skin and fat were synthesized as the skin-fat layer. The phantom model was a two layer model. The phantom experiments carried out in this article were consistent with the results of the FEM model. The Phantom model can be used as an effective supplement to the FEM model in the design and performance test of implantable transceiver, and the research of implantable channel in the future as well.

The study of the surface-to-implant signal transmission characteristics in this article was mainly focused on $\mathrm{cl}=6 \mathrm{~cm}$ and $\mathrm{cl}=10 \mathrm{~cm}$. For the actual BAN (body area network) application scenario, the implantable medical devices were distributed in all parts of the whole human body, and the channel length was likely to be longer than $10 \mathrm{~cm}$. Therefore, further studies on the surface-to-implant signal transmission characteristics of long distance are required. The author's future work is to establish the FEM model and the phantom model of the whole human body. On the basis of that, we will further explore the surface-to-implant signal transmission characteristics of the longer channel, which provides a theoretical basis for the development of the BAN.

\section{Conclusions}

In this article, we design an implantable FEM model and a phantom model using visible human leg data to study surface-to-implant implantable signal transmission characteristics in the frequency range from $10 \mathrm{kHz}$ to $1 \mathrm{MHz}$. Comparing the results, with an increase in frequency, surface-to-implant implantable signal attenuation remained basically unchanged, but with an increase in the channel length, signal attenuation increased. It can be concluded that in designing a recharger for implanted medical devices the channel length will be a key parameter. The mutual complementation and authentication of the FEM and phantom models lent veracity and reliability to the implantable signal transmission characteristics studied in this article. The results of this article may benefit the designing of rechargers and implantable transceivers for medical devices.

\section{Authors' contributions}

YMG contributed to conceived the study; YY wrote and edited the article; SL completed the determination of the results; MIV and MD conceived and designed the experiments; ŽLV and MC performed the experiments; SHP analyzed the data. All authors read and approved the final manuscript.

\section{Author details}

${ }^{1}$ College of Physics and Information Engineering, Fuzhou University, Fuzhou 3501 16, China. ${ }^{2}$ Key Lab of Medical Instrumentation \& Pharmaceutical Technology of Fujian Province, Fuzhou 350116, China. ${ }^{3}$ Faculty of Electrical Engineering and Computing, University of Zagreb, Zagreb, Croatia. ${ }^{4}$ State Key Laboratory of Analog and Mixed Signal VLSI, University of Macau, Macau 999078, China. ${ }^{5}$ Department of Electrical and Computer Engineering, Faculty of Science and Technology, University of Macau, Macau 999078, China.

\section{Acknowledgements}

This work was supported by the Project of Chinese Ministry of Science and Technology 2013DFG32530, the National Natural Science Foundation of China U1505251 and 61201397, the Project of the Department of Education of Fujian Province, China, JK20140001, and the 063/2009/A of FDCT Macau and RG072/0910S/MPU/FST Research Committee, University of Macau. The authors would like to express heartfelt thanks to U.S. National Library of Medicine and the University of Colorado for provided the transverse anatomical fault images of a male.

\section{Competing interests}

The authors declare that they have no competing interests. 


\section{Publisher's Note}

Springer Nature remains neutral with regard to jurisdictional claims in published maps and institutional affiliations.

Received: 28 February 2017 Accepted: 27 June 2017

Published online: 04 July 2017

\section{References}

1. Patel S, Park H, Bonato P, Chan L, Rodgers M. A review of wearable sensors and systems with application in rehabilitation. J Neuroeng Rehabil. 2012;9(1):1

2. Rodgers MM, Pai VM, Conroy RS. Recent advances in wearable sensors for health monitoring. Sens J IEEE. 2015;15(6):3119-26.

3. Ferguson JE, Redish AD. Wireless communication with implanted medical devices using the conductive properties of the body. Expert Rev Med Devices. 2011;8(4):427-33.

4. Moritz CT, Perlmutter SI, Fetz EE. Direct control of paralysed muscles by cortical neurons. Nature. 2008:456(7222):639-42

5. Wang XC, Zhang GH, Huo XL. Research progress of human body communication technology. Chin J Biomed Eng. 2015:34(3):345-53.

6. Lindsey DP, McKee EL, Hull ML, Howell SM. A new technique for transmission of signals from implantable transducers. IEEE Trans Biomed Eng. 1998:45(5):614-9.

7. Wegmueller MS, Huclova S, Froehlich J, Oberle M, Felber N, Kuster N, Fichtner W. Galvanic coupling enabling wireless implant communications. IEEE Trans Instrum Meas. 2009;58(8):2618-25.

8. Shiba K, Enoki N. Capacitive-coupling-based information transmission system for implantable devices: investigation of transmission mechanism. IEEE Trans Biomed Circuits Syst. 2013;7(5):674-81.

9. Ferguson JE, Redish AD. Wireless communication with implanted medical devices using the conductive properties of the body. Expert Rev Med Devices. 2011;8(4):427-33.

10. Tang Z, Sclabassi RJ, Sun C, Hackworth SA, Zhao J, Cui XT, Sun M. Transcutaneous battery recharging by volume conduction and its circuit modeling. Engineering in Medicine and Biology Society, 2006. EMBS'06. 28th Annual International Conference of the IEEE. New York: IEEE; 2006. p. 644-647.

11. Hackworth SA, Sun M, Sclabassi RJ. A prototype volume conduction platform for implantable devices. 2007 IEEE 33rd Annual Northeast Bioengineering Conference. 2007.

12. Zhou W, Liu W, Qiu Q, Liu R, Jiang L, Song R. Development, fabrication, and applications of biomedical electrodes. Chin Sci Bull. 2015;60(15):1352-60.

13. Song Y, Zhang K, Hao Q, Hu L, Wang J, Shang F. A finite-element simulation of galvanic coupling intra-body communication based on the whole human body. Sensors. 2012;12(10):13567-82.

14. Gao YM, Wu ZM, Vai MI, Vasić ŽL,Cifrek M. An investigation of the FEM simulation for the galvanic coupling IBC based on visible human data. 2015 IEEE International Conference on Consumer Electronics-China; 2015.

15. Gabriel S, Lau RW, Gabriel C. The dielectric properties of biological tissues: il. Measurements in the frequency range $10 \mathrm{~Hz}-20 \mathrm{GHz}$. Phys Med Biol. 1996;41(11):2251.

16. Liu ZY, Xing J, Jian Rk, Wang X. An analysis method of human body communication based on wearable device. Transducer Microsyst Technol. 2015;12:12.

17. Pun SH, Gao YM, Mak PU, Vai MI, Du M. Quasi-static modeling of human limb for intra-body communications with experiments. IEEE Trans Inf Technol Biomed. 2011;15(6):870-6.

18. Gao YM, Pun SH, Du M, Vai MI, Mak PU. A preliminary two dimensional model for intra-body communication of body sensor networks. International conference on intelligent sensors, sensor networks and information processing, 2008. ISSNIP 2008. New York: IEEE; 2008. p. 273-8.

19. Pun S H, Gao Y M, Mak P U, DuM, VaiMI. Modeling for intra-body communication with bone effect. Engineering in Medicine and Biology Society, 2009. EMBC 2009. Annual International Conference of the IEEE. New York: IEEE; 2009. p. 693-696.

20. Zeng ZX, Gao YM, Pun SH. Effects of muscle conductivity on signal transmission of intra-body communications. J Electron Meas Instrum. 2013;27(1):21-5.

Submit your next manuscript to BioMed Central and we will help you at every step:

- We accept pre-submission inquiries

- Our selector tool helps you to find the most relevant journal

- We provide round the clock customer support

- Convenient online submission

- Thorough peer review

- Inclusion in PubMed and all major indexing services

- Maximum visibility for your research

Submit your manuscript at www.biomedcentral com/submit 